\title{
EFEITOS DE TREINAMENTO NOS DESEMPENHOS INDIVIDUAL E ORGANIZACIONAL
}

\section{RESUMO}

O objetivo deste estudo foi desenvolver um método para identificar efeitos de treinamento nos desempenhos dos indivíduos e da organização, combinação ainda rara na literatura científica. Foi realizado no Banco do Brasil, com 218 profissionais que fizeram um MBA em Marketing. Os dados foram coletados por meio de entrevistas e questionários de auto e heteroavaliação. Nos questionários foi avaliada a contribuição do treinamento para a melhoria do desempenho individual e organizacional, e o suporte à transferência de treinamento. Nas entrevistas foram levantados indicadores de melhoria do desempenho organizacional para os maiores impactos percebidos pelos respondentes. Os resultados indicam que, para a maioria dos desempenhos individuais e organizacionais, o impacto percebido do treinamento foi avaliado entre bom e ótimo. A metodologia utilizada foi considerada adequada para a identificação de melhorias nos desempenhos organizacionais, percebidos como os mais afetados pelo treinamento. Também foram discutidas implicações metodológicas, teóricas e perspectivas futuras.

\section{Isa Aparecida de Freitas}

UnB e Universidade Corporativa Banco do Brasil

\section{Jairo Eduardo Borges-Andrade}

UnB

\begin{abstract}
This study's objective was to develop a method for identifying training effects on performance, at individual and organizational levels, a still rare combination in the scientific literature. The study was made at Banco do Brasil, with 218 MBA in Marketing ex-trainees. Data collection included questionnaires for self and third party assessments and interviews. In the questionnaires, respondents have assessed the contribution of training for individual and organizational performance improvements, and organizational support for training transfer. In the interviews, indicators of organizational performance improvements were identified, based on the highest impacts perceived by the respondents. The results demonstrate that the perceived impact of training, for most part of individual and organizational performances, was considered between good and great. The methodology used was adequate to identify organizational performances improvement noticed as most affected by the training. Methodological and theoretical implications and future perspectives are discussed.
\end{abstract}

PALAVRAS-CHAVE Avaliação do treinamento, resultados do treinamento, impacto do treinamento no desempenho individual e na organização, treinamento em Marketing.

KEY WORDS Training evaluation, results of training, impact of training on individual work, impact of training on the organization, marketing training. 


\section{INTRODUÇÃo}

Há uma crescente preocupação entre os pesquisadores e os profissionais acerca da avaliação de treinamento. Isso é devido, principalmente, ao aumento do volume de investimentos em treinamento pelas organizações e à necessidade de mensuração de sua eficácia, tanto no nível individual quanto no organizacional. $\mathrm{Na}$ avaliação de treinamento, são colhidas informações sistemáticas sobre todo o processo, e é atribuído valor ao treinamento, com o julgamento do grau em que ele contribuiu para o desempenho dos indivíduos, grupos e organização. A avaliação também funciona por meio da realimentação do sistema de treinamento, ao identificar necessidades de aperfeiçoamento nos programas e ao apontar os aspectos positivos. Um dos seus desafios é verificar as contribuições desses programas em termos de seus efeitos em níveis distintos.

Este artigo tem como objetivo descrever uma metodologia de avaliação de treinamento desenvolvida para identificar tais efeitos nos desempenhos dos indivíduos e da organização. Também pretende contribuir para o enriquecimento do tema, pois como foi recentemente diagnosticado por Borges-Andrade (2002), estudos que envolvem a avaliação da contribuição do treinamento para a organização são pouco freqüentes nas literaturas brasileira e estrangeira.

\section{AVALIAÇÃO DO TREINAMENTO NO NÍVEL ORGANIZACIONAL}

Avaliar treinamento não é uma preocupação recente. Um modelo clássico é o sugerido por Donald Kirkpatrick (1976), que define quatro níveis de avaliação: reações, aprendizagem, comportamento no cargo e resultados. As reações representam medidas de satisfação com o treinamento em termos de qualidade do programa, instalações e desempenho do instrutor. A aprendizagem diz respeito à avaliação das diferenças de desempenho dos treinandos antes e depois do treinamento, em termos dos objetivos instrucionais. O comportamento no cargo aborda as melhorias no desempenho do treinando no trabalho, decorrentes da aplicação do que foi aprendido no treinamento. Os resultados enfocam as mudanças ocorridas no nível da organização em função do treinamento.

Um estudo realizado por Jack Philips (1997) nas empresas americanas indicou que $100 \%$ das organizações avaliavam o treinamento no nível de reações, $40 \%$ avaliavam a aprendizagem ao final do treinamento, $16 \%$ avaliavam o comportamento no cargo, e somente $4 \%$ avaliavam a contribuição do treinamento para a organização. Segundo o autor, esses percentuais podem estar associados às dificuldades metodológicas em identificar resultados de treinamento no nível macro, bem como à necessidade de recursos financeiros e de tempo para a realização de avaliações dessa natureza.

No Brasil, já foram realizados estudos regionais ou setoriais, mas inexistem estudos amplos e nacionais publicados que identifiquem dados quantitativos referentes ao que as organizações avaliam em cada nível. Em termos de disponibilidade tecnológica, BorgesAndrade (2002) ressaltou a ocorrência de avanços na elaboração de medidas para mensurar reações e comportamento no cargo. Destacou também a necessidade de mais esforços para a elaboração de medidas de aprendizagem e enfatizou a necessidade de sistematização de medidas para o nível de resultados.

Desde a década de 1980, muitos modelos referentes à produção do conhecimento em avaliação do treinamento foram formulados e testados, tais como os de Borges-Andrade (1982), Noe e Schmitt (1986), Rouillier e Goldstein (1993), Abbad (1999), Sallorenzo (2000), Lacerda e Abbad (2003), Meneses e Abbad (2003). Apesar do crescente interesse nesse tema, tais modelos abordaram basicamente os preditores de impacto de treinamento no desempenho do indivíduo. Adicionalmente, o presente artigo focaliza também os impactos do treinamento nos níveis do indivíduo e da organização, conforme descritos a seguir.

Impacto de treinamento no trabalho se refere ao terceiro nível de avaliação proposto por Donald Kirkpatrick (1976) - denominado comportamento no cargo - e pode ser conceituado como a ocorrência de melhorias significativas no desempenho específico (impacto em profundidade) ou no desempenho geral da pessoa treinada (impacto em amplitude), como resultado da aplicação no trabalho dos conhecimentos, habilidades e atitudes (CHAs) aprendidos em treinamento. Esses dois tipos de impacto têm sido avaliados pelos pesquisadores, e os resultados indicaram a existência de correlação positiva e significativa entre essas medidas (Borges-Andrade, 2002).

Na revisão de literatura realizada por Abbad, Pilati e Pantoja (2003) foi analisada a produção nacional e a estrangeira no período de 1998 a 2001 acerca dos preditores de impactos de treinamento. Constatou-se que os estudos indicaram variáveis antecedentes do impacto do treinamento no trabalho distribuídas em 
três categorias: características individuais, do treinamento e de suporte ou clima para transferência. Quanto às características do indivíduo, destacaram-se como preditoras: comprometimento no trabalho, motivação para treinamento, idade, auto-eficácia e locus de controle. As duas últimas variáveis são relativas à personalidade do treinando. Auto-eficácia refere-se à percepção do indivíduo quanto à sua capacidade para obter sucesso em suas realizações, e locus de controle refere-se à crença do indivíduo nos fatores internos ou externos que controlam os eventos e o seu próprio comportamento. Quanto às características dos treinamentos, os de natureza cognitiva apresentaram maiores índices de impacto do que os afetivos, havendo poucos estudos sobre os psicomotores. A revisão indica também que o preditor mais pesquisado foi o suporte à transferência, sendo os principais antecedentes de impacto de treinamento no desempenho do indivíduo o suporte psicossocial ou o apoio dado pelos chefes e colegas à aplicação no trabalho dos CHAs aprendidos. Na literatura estrangeira essa variável é muitas vezes denominada "clima para transferência". Apesar das diferenças metodológicas encontradas nas pesquisas realizadas no Brasil e no exterior, existem resultados similares, principalmente quanto à importância do suporte à transferência na predição de impacto de treinamento.

De acordo com a revisão feita por Sallas e CannonBowers (2001) da produção internacional sobre treinamento e desenvolvimento no período de 1992 a 2001, a área de treinamento já adquiriu o status de ciência devido à atual robustez do escopo teórico a partir dos resultados empíricos das pesquisas. Esses resultados podem subsidiar a tomada de decisão nas organizações, pois já existem metodologias para identificar o impacto de treinamento no trabalho e de mensuração das variáveis a ele relacionadas, além de técnicas para aumentar a transferência de treinamento, como as propostas por Broad e Newstrom (1992). As pesquisas de avaliação de treinamento investigaram os efeitos da aprendizagem no desempenho do aprendiz (transferência horizontal). Contudo, pouco se investigaram os efeitos no grupo, na unidade de trabalho e na organização.

Alguns manuais, como o de Phillips (1997), sugerem o uso do ROI (retorno sobre investimentos) como a metodologia ideal a ser utilizada para esse último tipo de avaliação. Ele sugeriu coletar dados sobre os dois aspectos da equação de ROI: os custos do treinamento e o que ele gerou de resultado financeiro para a organização. A primeira parte da equação é simples de calcular. Basta ter um registro sistemático dos custos com a produção e execução do treinamento, tais como despesas com o planejamento, instrutores, tutores, passagens, hospedagem e produção de material. As dificuldades ocorrem quando se tenta mensurar o valor financeiro da contribuição do treinamento e isolar as outras variáveis organizacionais que também contribuem para os resultados. A despeito dessas dificuldades, Ávila et al. (1983) desenvolveram o que parece ser o único trabalho brasileiro sobre avaliação de impacto de treinamento na organização em termos de ROI. Mas é necessário ainda desenvolver metodologias capazes de captar resultados organizacionais em outros termos. Além disso, Borges-Andrade (2002) destacou que o uso pouco freqüente da metodologia ROI deve-se à complexa combinação de habilidades específicas necessárias para a realização desse trabalho, do tempo despendido e do custo, inclusive político, embutidos na sua consecução.

Dessa forma, avaliar treinamento no nível da organização é mais abrangente que utilizar o ROI, por ser necessário mensurar outras mudanças organizacionais ocorridas em função do treinamento, tais como efeitos nos processos de trabalho, na produtividade, no clima e na cultura da organização.

Birdi (2000) propôs uma taxonomia em vários níveis para classificar os possíveis efeitos do treinamento. Para esse autor, os treinamentos podem gerar efeitos nos níveis do indivíduo, da equipe e da organização. Em função do objetivo do artigo, serão destacados os efeitos no nível da organização. Tais efeitos são relativos a: aquisição de recursos como, por exemplo, novos clientes; compra de outras organizações; processos internos, em termos da adoção de novas tecnologias; clima organizacional; absenteísmo, rotatividade e taxas de acidentes de trabalho; metas de produtos, referentes à quantidade, qualidade e variedade da produção; metas do sistema, em termos de crescimento e lucro da organização, ROI, imagem organizacional, satisfação de consumidores e acionistas.

A abordagem multinível proposta por Klein e Kozlowski (2000) também contribui para a avaliação de treinamento no nível da organização. Esses autores ressaltam que as organizações são sistemas multiníveis, onde o nível inferior (indivíduo) está contido nos níveis mais abrangentes (grupo e organização). Por isso, os fenômenos que ocorrem nas organizações podem ser explicados por variáveis de diferentes níveis, tais como segmentos de interesse teórico - que represen- 
tam grupamentos humanos -, duplas, grupos, unidades e organização (Puente-Palacios, 2003). Nesse sentido, os níveis são definidos de acordo com o fenômeno a ser investigado pelo pesquisador. Por exemplo, o impacto de treinamento no desempenho do indivíduo pode ser em decorrência do próprio indivíduo (motivação), do grupo ao qual ele pertence (tamanho, qualidade da interação) e do tipo de organização (pública ou privada). A Figura 1 ilustra os dois tipos de influência tratados na abordagem multinível.

De acordo com essa abordagem, tanto as organizações podem influenciar os comportamentos dos indivíduos quanto os indivíduos podem modificar os processos organizacionais. A primeira direção da influência é denominada processos "topo-descida", e a segunda se refere aos processos "base-subida". O impacto de treinamento no nível da organização é um fenômeno "base-subida", pois é o indivíduo quem é treinado. Espera-se que o desempenho individual seja aperfeiçoado e que gere mudanças no grupo de trabalho e na organização. Segundo Klein e Kozlowski (2000), os fenômenos "base-subida" são mais lentos que os "topodescida", necessitando de mais tempo para serem percebidos pelos pesquisadores. Embora a presente pesquisa não tenha um desenho multinível, foi inspirada nessa abordagem, principalmente na busca de indicadores adequados dos níveis de desempenho individual e organizacional.

Figura 1 - Abordagem multinível.

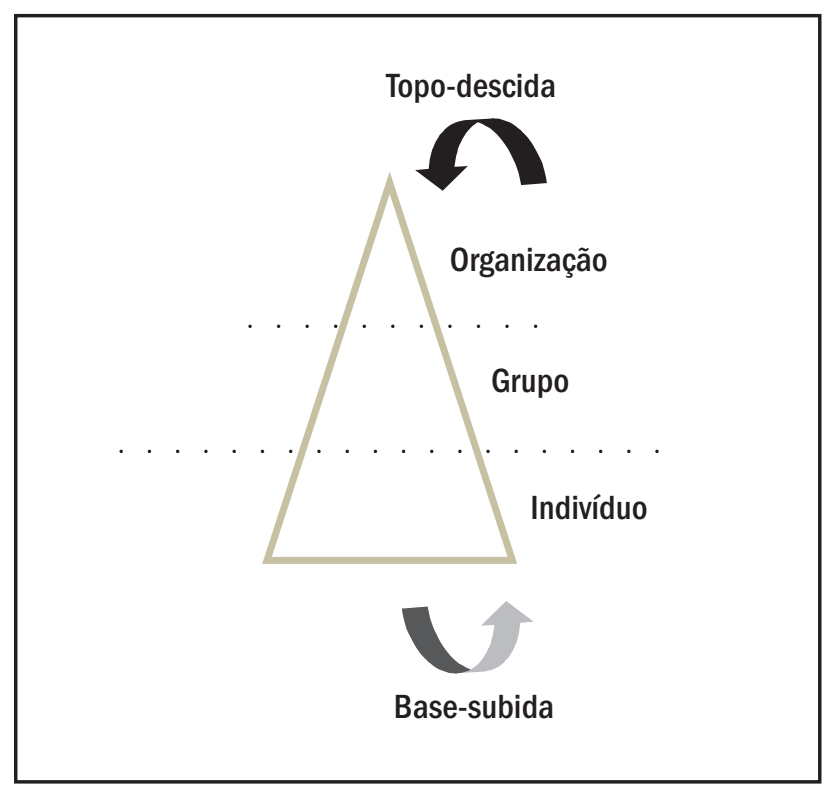

Fonte: Adaptado de Puente-Palacios (2003).

\section{MÉTODO}

O treinamento utilizado para a aplicação do método de avaliação desenvolvido foi o BB MBA Marketing, curso de especialização lato sensu, de modalidade presencial, com 360 horas distribuídas em 20 disciplinas, desenvolvido em parceria com duas diferentes instituições de ensino de renome. Algumas disciplinas tiveram avaliações de aprendizagem em grupo. Os objetivos de aprendizagem foram diversificados em cada uma dessas disciplinas. Como resultados esperados no desempenho dos funcionários treinados, estavam a criação, a implementação e a avaliação de ações e procedimentos voltados para a atração e manutenção dos clientes da empresa.

A população da pesquisa foi formada por 218 técnicos que atuavam em diversas unidades do Banco do Brasil. Esses profissionais participaram do curso no período de 1996 a 2000, compondo sete turmas, com média de 31 participantes em cada uma. Os questionários foram elaborados seguindo os procedimentos adotados por Borges-Andrade e Siri (2000), com adaptações que levaram em conta as características do treinamento estudado. Foram remetidos questionários aos 199 treinados que permaneceram na empresa e que haviam concluído o treinamento há no mínimo um ano, obtendo-se um retorno de 94 questionários.

A investigação do impacto do BB MBA Marketing no trabalho dos indivíduos centrou-se no efeito dos conteúdos apreendidos nos desempenhos específicos, ou avaliação em profundidade. Esses desempenhos específicos (27 itens) foram construídos a partir da análise do material instrucional do curso e validados semanticamente por um conjunto de 10 participantes do treinamento.

Segundo a percepção dos funcionários, a investigação do impacto no nível da organização se centrou na busca da contribuição do treinamento para a empresa. Para a construção dos itens, foram utilizadas análises do material didático, das "notas" para contratação do treinamento, e quatro entrevistas com representantes das principais unidades do banco. O objetivo da entrevista foi identificar as mudanças ocorridas nas unidades que pudessem ser vistas como contribuições do treinamento. No total, foram construídos 26 itens, que passaram por um processo de validação semântica realizada com 13 representantes das unidades dos treinados.

O instrumento de coleta de dados respondido pelos 
treinados para a auto-avaliação foi composto por questões fechadas e abertas, divididas em 5 partes: a) dados demográficos; b) avaliação da necessidade e do grau de utilização da aprendizagem no trabalho, utilizando-se uma escala de concordância de 1 a 6 pontos, na qual $1=$ "discordo totalmente" e $6=$ "concordo totalmente". Adicionalmente foram solicitadas as razões da não utilização; c) percepção sobre o impacto de treinamento no trabalho, tendo os 27 itens sido avaliados com uma escala de 10 pontos, na qual 1 = "nenhuma contribuição" e 10 = "contribuição muito grande"; d) percepção sobre suporte à transferência de treinamento, formado por 10 itens que foram avaliados com uma escala de concordância de 10 pontos, na qual 1 = "não concordo" e $10=$ "concordo totalmente"; e) percepção sobre o impacto de treinamento no desempenho da organização, formada por 26 itens, avaliada com a mesma escala utilizada na parte "c".

O questionário de heteroavaliação foi composto pelas partes "c" e "e" do questionário de auto-avaliação, e respondido pelos pares ou pelo superior imediato do indivíduo treinado. Buscou-se maior fidedignidade dos resultados apresentados com o uso de dois diferentes tipos de fontes. Obteve-se um retorno de 57 questionários.

Depois da análise dos resultados dos questionários de auto e heteroavaliação sobre impacto de treinamento na organização, foram realizadas 10 entrevistas estruturadas - individuais e coletivas - com representantes de diversas unidades da empresa. O objetivo foi verificar a existência de indicadores organizacionais que confirmassem ou não as percepções individuais dos funcionários sobre os maiores impactos do curso no nível da organização. Para isso foram utilizados os 10 primeiros itens com os maiores impactos, e acrescidos dois itens extraídos dos propósitos de marketing, que focam a capacidade da empresa de atrair e manter clientes. A escolha das unidades foi feita com base na identificação das áreas responsáveis pela gestão de cada um dos desempenhos organizacionais aperfeiçoados. Nessas entrevistas foram repassados os objetivos da pesquisa e os principais resultados do BB MBA Marketing para a organização, no ponto de vista dos treinados. Pediu-se aos entrevistados que listassem indicadores organizacionais que confirmassem ou não esses resultados. Além disso, levantaram-se outros possíveis resultados do MBA e outros fatores organizacionais que contribuíram para a concretização dos resultados identificados.

\section{RESULTADOS E DISCUSSÃO}

Os resultados da pesquisa apresentados nesta seção estão organizados em tópicos. Cada resultado será apresentado e imediatamente discutido.

\section{Características da clientela}

Os dados demográficos da clientela estudada indicaram que a maioria dos treinados era do sexo masculino $(60 \%)$, possuíam no mínimo pós-graduação (54\%), com formação em diversas áreas; atuavam como técnicos (70\%); trabalhavam na administração central (98\%), em unidades diversificadas; e apresentavam tempo de função entre um e três anos (56\%). Esses profissionais eram responsáveis pela formulação de estratégias e políticas para a criação de novos produtos e serviços voltados para clientes externos e internos.

\section{Impactos do treinamento no desempenho dos indivíduos}

De acordo com as respostas dadas à questão "utilizo no meu trabalho os conteúdos aprendidos no treinamento", foram identificados dois grupos de respondentes. O primeiro são os treinados que não utilizaram a aprendizagem (assinalaram 1 e 2 na escala de concordância), e o segundo são aqueles que marcaram de 3 a 6 (na mesma escala), evidenciando utilizar o aprendido. A grande maioria (81 a 93\%) dos respondentes relatou que na situação de trabalho aplicava as aprendizagens adquiridas no curso. Os seis funcionários que não utilizaram os conteúdos aprendidos destacaram que suas atividades atuais não exigiam a aplicação do que aprenderam. Neste artigo serão apresentados apenas os resultados apontados pelos funcionários que aplicaram a aprendizagem no trabalho.

Foram comparadas as percepções dos treinados (87) com as percepções de 57 superiores imediatos ou colegas desses profissionais. As médias de impacto situaram-se entre 7,39 e 4,62 na auto-avaliação e entre 8,44 e 5,37 para a heteroavaliação. Embora em magnitude a heteroavaliação tenha sido mais favorável, a análise pareada de dados mostrou que não há diferença estatisticamente significativa entre as avaliações feitas por essas duas fontes, no nível de significância de 0,05.

Tal verificação dá suporte a achados de outros autores, mencionados anteriormente, nos quais a auto e heteroavaliação convergem para as mesmas conclusões. Tanto os estudos anteriormente mencionados quanto o presente utilizaram treinamentos cujo foco central são "conhecimentos" e "habilidades". Em casos de trei- 
namentos focados em "atitudes", as auto-avaliações tendem a ser lenientes.

Com base nos dados da heteroavaliação, classificaram-se os 27 desempenhos específicos pelo grau de impacto: $15 \%$ dos desempenhos (4) obtiveram ótimos impactos, 59\% dos desempenhos (16) apresentaram bons impactos e $26 \%$ dos desempenhos (7) tiveram impactos regulares. Os resultados das duas fontes, para os cinco maiores impactos em desempenhos específicos, estão descritos na Tabela 1. Os maiores impactos ressaltaram a melhoria em desempenhos voltados para a valorização do cliente.

Para verificar as relações entre as variáveis da clientela (nível de escolaridade, formação, local de trabalho, cargo e tempo na função) e a percepção de aplicação das aprendizagens, foram realizadas análises de variância e constatou-se que não há diferença estatística significativa utilizando-se o nível de significância de 0,05 . Portanto, as características demográficas, funcionais e ocupacionais não demonstraram ser preditoras de impacto no nível individual, o que igualmente confirma resultados de outros estudos. Resta investigar a relação entre o suporte à transferência e o impacto de treinamento.

\section{Suporte à transferência}

Para a validação estatística da escala de suporte à transferência foi utilizada a análise de componentes principais, análise fatorial (PAF, com rotação oblíqua), substituição dos casos faltosos pela média e análise de confiabilidade, por meio do Alpha de Cronbach. A análise de componentes principais, utilizando a escala de 10 itens, identificou que a matriz era fatorável (a relação número de casos por item foi de 8,1 , com $\mathrm{KMO}=0,87$, determinante igual a 2,488 E-04). Essas análises identificaram uma estrutura com dois fatores, correlaciona- dos entre si, que explicam $72 \%$ da variância das respostas da escala. Esses dados são muito semelhantes aos relatados por outros autores, tais como Abbad (1999), Sallorenzo (2000), Lacerda e Abbad (2003), Meneses e Abbad (2003), que utilizaram essa mesma escala.

A análise do conteúdo dos itens do fator 1 revelou que se trata de suporte psicossocial, incluindo conteúdos relativos ao apoio dos colegas e chefes para a aplicação do aprendido. Esse fator contém sete itens, com Alpha de Cronbach de 0,92 e percentual de variância explicada de $63 \%$. Obteve média de 7,85 e desvio padrão de 0,6 , numa escala de concordância de 1 a 10. O fator 2 incluiu itens sobre suporte material relativos à disponibilidade de recursos e de informações necessárias à aplicação da aprendizagem, além de espaço físico adequado. Esse fator é formado por três itens, com Alpha de Cronbach de 0,85, e apresentou percentual de variância explicada de $10 \%$. A média obtida no fator 2 foi de 7,0 com desvio padrão de 0,5 . Foram replicados, assim, os excelentes indicadores psicométricos desses fatores da escala de suporte à transferência, bem como os constructos a eles subjacentes, encontrados nos estudos mencionados anteriormente.

As médias obtidas nos dois fatores indicaram que as pessoas percebiam a presença de suporte à aplicação no trabalho das novas aprendizagens. Os valores relativos aos desvios padrão sugerem que os participantes possuem percepções homogêneas quanto aos aspectos avaliados. A análise de variância mostrou que o fator relativo ao suporte psicossocial apresenta relações positivas e significativas com o nível de aplicação do treinamento. Não foi detectada relação para o suporte material, demonstrando que esse aspecto não se constitui num diferencial que gere um impacto mais elevado.

Tabela 1 - Impactos mais elevados nos desempenhos específicos, em ordem decrescente.

\begin{tabular}{|c|c|c|c|c|}
\hline \multirow{2}{*}{ DESEMPENHOS INDIVIDUAIS ESTUDADOS } & \multicolumn{2}{|c|}{ HETEROAVALIAÇÃO } & \multicolumn{2}{|c|}{ AUTO-AVALIAÇÃO } \\
\hline & MÉDIA & DP & MÉDIA & DP \\
\hline $\begin{array}{l}\text { Utilizar informações de pesquisas para apoiar a tomada de } \\
\text { decisão em marketing, visando obter ou manter vantagem competitiva. }\end{array}$ & 8,44 & 2,12 & 7,38 & 2,72 \\
\hline Realizar negociações de forma organizada, consciente e estruturada. & 8,44 & 1,79 & 7,79 & 1,98 \\
\hline $\begin{array}{l}\text { Desenvolver ações visando um serviço de excelência, com base } \\
\text { na ótica do cliente. }\end{array}$ & 8,28 & 2,50 & 7,74 & 2,64 \\
\hline Avaliar a qualidade dos serviços com base na satisfação do cliente. & 8,28 & 2,51 & 7,22 & 2,78 \\
\hline $\begin{array}{l}\text { Propor estratégias de marketing de relacionamento, visando } \\
\text { satisfação e fidelização do cliente. }\end{array}$ & 8,14 & 2,51 & 7,46 & 2,79 \\
\hline
\end{tabular}


A importância do suporte à transferência, principalmente o suporte psicossocial, tem sido confirmada nas diversas pesquisas realizadas nos últimos 20 anos, tanto nos estudos nacionais como nos estrangeiros, como pode ser visto na revisão de literatura feita por Abbad, Pilati e Pantoja (2003). Portanto, o suporte psicossocial deveria ser o aspecto fundamental a ser considerado pelos gestores interessados em melhorar os efeitos desse treinamento no desempenho dos indivíduos treinados. Contudo, tais efeitos representam simplesmente uma transferência horizontal. Entrevistas e inspeção nos documentos que justificaram o treinamento, avaliado no presente trabalho, explicitavam que a empresa esperava muito mais do que mudanças em desempenhos individuais. Como verificar os efeitos no nível da organização? A próxima seção tratará desse assunto.

\section{Impactos do treinamento na organização}

Os 26 itens relativos ao impacto na organização foram avaliados pelos funcionários treinados e seus superiores imediatos ou colegas. A avaliação ocorreu no primeiro semestre de 2002. Assim, houve um intervalo de tempo que variou de um a cinco anos após o término do treinamento. O tempo mínimo de um ano foi utilizado para possibilitar que os efeitos do treinamento na organização pudessem ser observados.

As médias de impacto do treinamento na organização situaram-se entre 8,78 e 6,38 na heteroavaliação e entre 8,08 e 5,21 na auto-avaliação (escala de 10 pontos). Por meio de análise de variância foram constatadas diferenças significativas entre a auto-avaliação e a heteroavaliação em quatro itens, considerando-se o nível de significância de 0,05. Nesses casos, as médias de impacto foram maiores nas avaliações feitas pelos superiores ou colegas dos indivíduos treinados, resultado similar ao obtido por Borges-Andrade e Siri (2000), que atribuíram o ocorrido a um forte componente ideológico do treinamento por eles estudado, que teria tornado os indivíduos treinados muito mais conservadores que os demais em relatar o efetivo uso no trabalho do aprendido em treinamento. No presente caso, uma possível explicação para o fato é que, por se tratar de segmento estratégico, os superiores podem ter acesso a informações diferenciadas, que pode ter auxiliado na percepção de um maior impacto nos níveis superiores da organização.

Dos 26 desempenhos organizacionais considerados na heteroavaliação, $31 \%$ obtiveram ótimo impactos (8), $65 \%$ apresentaram bons impactos (17) e apenas um teve impacto regular (4\%). As opiniões sobre o impacto na organização foram muito convergentes, tal como já foi verificado nas avaliações de impacto no desempenho dos indivíduos. Da lista dos 10 maiores impactos, para os dois grupos, sete foram comuns.

Com base nesses resultados, foram realizadas entrevistas estruturadas com o objetivo de investigação intensiva, para verificar se as percepções no nível individual, colhidas por meio de questionários, podiam ser confirmadas ou não por outros indicadores organizacionais e usando outro método de coleta de dados. O Quadro 1 apresenta os 12 desempenhos e seus indicadores.

Os desempenhos organizacionais descritos no Quadro 1 serão apresentados, de forma sintética, a seguir. Adicionalmente são feitas algumas considerações sobre os seus indicadores. Só foram considerados os indicadores obtidos a partir de 1997, um ano após o término da primeira turma do curso.

\section{Elevação do nível de competência em marketing e aumento da credibilidade técnica do marketing do BB}

Devido à proximidade conceitual que os dois desempenhos organizacionais apresentam, foram buscados indicadores que atendessem a ambos. A credibilidade técnica do marketing tanto pode ser atribuída internamente quanto externamente, e a elevação no nível de competência segue o mesmo parâmetro. Dois indicadores foram identificados: os prêmios em marketing recebidos e a melhoria na qualidade das demandas internas de marketing.

No que se refere aos prêmios, podem ser destacados os quatro intitulados Prêmio Destaque no Marketing recebidos da Associação Brasileira de Marketing e Negócios, nos anos de 1997, 2000 e 2001, nas categorias "Cultura, Lazer e Entretenimento", "Terceiro Setor" e "Marketing Promocional". A empresa recebeu também um Prêmio Marketing Best, concedido pela Media Mundo Marketing, Fundação Getúlio Vargas e Editora Referência, que é considerado um dos mais importantes prêmios de marketing do país, pela campanha "BB.com.br [e-pronto]".

No que se refere à qualidade das demandas internas, destinadas à área de marketing, os técnicos entrevistados destacaram que as demandas são mais completas, com objetivos vinculados à estratégia organizacional, e descreveram com mais precisão os objetivos da comunicação, o público-alvo e até os canais mais apropriados. Isso foi possível com a criação de um 
"modelo de brieffing", que auxilia na solicitação dessas demandas.

\section{Maior disseminação dos conteúdos de marketing na organização}

$O$ indicador identificado para esse item foi a quantidade de treinamentos internos desenvolvidos sobre o tema marketing, de funcionários treinados nesses cursos, e de instrutores formados. A primeira turma do curso ocorreu em 1996, e os dados foram coletados em junho de 2002. Nesse período, a quantidade de pessoas treinadas correspondeu a 61.225, o que representa $80 \%$ dos funcionários da empresa. É importante ressaltar que do total de 157 instrutores formados para ministrar esses treinamentos, 54\% (85) são funcionários treinados no curso avaliado. Eles também atuam como especialistas de conteúdos dessa área na elaboração dos treinamentos internos.

\section{Melhoria da comunicação do BB com os clientes}

Nesse item foram pesquisados indicadores da percepção do cliente e de especialistas quanto à melhoria da comunicação. Além disso, verificaram-se as principais ocorrências e a satisfação do cliente com o BB Responde, veículo de comunicação direta do banco com os clientes. Quanto ao cliente interno, buscaram-se indicadores de satisfação com o RH Responde, canal de comunicação direta dos funcionários com a Diretoria de Gestão de Pessoas, criado em 2000 por um grupo de ex-treinandos do curso.

No que se refere à percepção dos clientes e de especialistas em comunicação e marketing, pode-se destacar o recebimento de 48 prêmios no período de 1997 a 2002. Esses prêmios envolveram várias categorias, tais como marketing esportivo, social, cultural e promocional, marca mais lembrada (Top of Mind), desempenho em produtos e serviços, tecnologia da informação, e Internet. O prêmio internacional foi o Best Corporate/Institutional Bank, promovido pela revista norte-americana Global Finance Magazine, na categoria Brazil.

$\mathrm{Na}$ avaliação do BB Responde constatou-se que os clientes estão mais satisfeitos com o atendimento prestado, considerando-se os índices de satisfação em 1997 ( $76 \%$ dos clientes) e 2001 ( $87 \%$ dos clientes satisfeitos). Houve também um aumento do número de ligações anuais da ordem de 510\% no período de 1998 a 2001, o que representa a confiança do cliente em relação às informações prestadas pelo canal. No período de 2001 ao primeiro semestre de 2002 as consultas mais freqüentes foram relativas aos produtos e serviços. Em relação ao número de reclamações, houve uma diminuição significativa (de $8 \%$ para $4 \%$ ) no mesmo período.

Quadro 1 - Indicadores de melhoria do desempenho organizacional como resultado do treinamento.

\begin{tabular}{|c|c|}
\hline DESEMPENHOS ORGANIZACIONAIS & INDICADORES DE MELHORIA (A PARTIR DE 1997) \\
\hline $\begin{array}{l}\text { - Elevação da competência em marketing } \\
\text { - Aumento da credibilidade técnica do marketing }\end{array}$ & $\begin{array}{l}\text { - Recebimento de } 5 \text { prêmios em marketing } \\
\text { - Melhoria na qualidade das demandas internas }\end{array}$ \\
\hline - Disseminação dos conteúdos de marketing & $\begin{array}{l}\text { - Elaboração de } 5 \text { cursos internos de marketing } \\
\text { - Formação de } 157 \text { instrutores } \\
\text { - Treinamento de } 61.225 \text { pessoas nos } 5 \text { cursos }\end{array}$ \\
\hline - Melhoria da comunicação com os clientes & $\begin{array}{l}\text { - Recebimento de } 48 \text { prêmios } \\
\text { - Aumento da satisfação com o BB Responde e com o RH Responde }\end{array}$ \\
\hline $\begin{array}{l}\text { - Aumento da competência em marketing, em relação a } \\
\text { outras estatais e a bancos privados }\end{array}$ & · Não há indicador \\
\hline - Melhoria da imagem institucional & · Não há indicador \\
\hline - Melhoria no desenvolvimento de produtos e serviços & - Processos mais focados no cliente \\
\hline - Melhoria da satisfação do cliente & $\begin{array}{l}\text { - Aumento de } 82 \% \text { no número de pessoas físicas muito satisfeitas } \\
\text { com o BB (1999 a 2001) }\end{array}$ \\
\hline - Aumento da competência técnica em benchmarketing & - Criação do Programa de Arquitetura de Informações \\
\hline - Aumento da competência técnica em pesquisa aplicada & - Criação de 4 tipos de pesquisa de satisfação do cliente \\
\hline - Aumento da base de clientes & - Acréscimo de 119\% de clientes Pessoa Física e 21 \% de Pessoa Jurídica \\
\hline - Diminuição da perda de clientes & · Não há indicador \\
\hline
\end{tabular}


O RH Responde foi apontado nas entrevistas como a principal ação de endomarketing criada após o treinamento. Em maio de 2002, mais de 85\% das consultas foram respondidas no mesmo dia. Em 2000 cerca de $25 \%$ das pessoas não se identificavam, preferindo o anonimato. Em maio de 2002, esse percentual esteve em torno de $1 \%$. Isso pode significar que os funcionários têm mais confiança para expressar suas opiniões sobre os programas de RH e sentem-se mais ouvidos. A Tabela 2 apresenta os resultados da pesquisa realizada para avaliar características importantes para esse tipo de canal. Esses resultados mostram que o RH Responde tem atendido aos requisitos exigidos e melhorado esses índices ao longo do tempo.

Em síntese, os resultados apresentados confirmam a percepção dos treinados de que houve melhoria na comunicação do Banco com os clientes externos e internos.

\section{Aumento da competência em marketing do BB em relação a outras empresas - estatais e bancos privados}

Não houve acesso a dados que permitissem a comparação direta do desempenho do banco com empresas estatais e com bancos privados. Portanto, não houve evidências que comprovassem a melhoria desse desempenho.

\section{Melhoria da imagem institucional decorrente da adoção de estratégias de marketing}

A primeira experiência na investigação da imagem institucional foi realizada em 2001 por meio da inclusão de um fator na Pesquisa de Satisfação do Cliente. Os resultados dessa pesquisa indicaram que tanto os clientes Pessoa Física (81\%) quanto as micro e pequenas empresas $(67,5 \%)$ estavam satisfeitas com a imagem da empresa no mercado. Não existem outros dados que possam indicar se ocorreu melhoria ou não da imagem institucional.

\section{Melhoria no desenvolvimento de produtos e serviços utilizando análise da concorrência e do mercado}

Pelo fato de o desempenho organizacional tratar do processo de desenvolvimento de produtos e serviços não foi possível precisá-lo em termos quantitativos. Os dados de entrevista revelaram que a preocupação com a atuação da concorrência na formulação de produtos e serviços é cada vez maior. Os técnicos acompanham os produtos e serviços desenvolvidos pela concorrência e suas características, benefícios e preços, o que permite criar produtos e serviços mais competitivos.

\section{Melhoria da satisfação do cliente com o BB}

Foram realizadas duas pesquisas sobre a satisfação do cliente com o banco, uma para cliente Pessoa Física e outra para Pessoa Jurídica. A pesquisa relativa à satisfação do primeiro segmento começou a ser realizada em 1999, e o índice de clientes muito satisfeitos aumentou de $17 \%$ para $28 \%$. Esse índice superou a meta definida pelo banco, que era ter $20 \%$ desses clientes muito satisfeitos ao final de 2001. Em termos de Pessoa Jurídica, houve apenas uma pesquisa em 2001. No que se refere à satisfação da Pessoa Física, os resultados encontrados corroboram com as percepções dos funcionários treinados de que aumentou a satisfação do cliente.

\section{Aumento da competência técnica do BB em benchmarking}

A evidência da melhoria desse desempenho organizacional foi a criação do Programa de Arquitetura de Informações - PAI. Esse programa estrutura e integra as informações da organização, a atuação dos concorrentes em diversas áreas, os indicadores financeiros, os programas e projetos da empresa. Ele facilita a identificação das informações mais importantes da concorrência para uma determinada finalidade. Nesse senti-

Tabela 2 - Comparação dos requisitos avaliados no RH Responde.

\begin{tabular}{|l|c|c|}
\hline \multicolumn{1}{|c|}{ QUESITOS AVALIADOS } & ANO 2000 & ANO 2001 \\
\hline Clareza e objetividade das informações & $89,4 \%$ & $92 \%$ \\
\hline Confiabilidade das informações & $90,8 \%$ & $93 \%$ \\
\hline Cordialidade do atendente & $98,5 \%$ & $100 \%$ \\
\hline Interesse pelo questionamento & $96,3 \%$ & $95 \%$ \\
\hline Segurança na condução & $89,2 \%$ & $92 \%$ \\
\hline Indicação do canal para outros colegas & $94,3 \%$ & Não avaliado \\
\hline
\end{tabular}

Fonte: Banco do Brasil, 2002. 
do, sua criação confirma a visão dos funcionários de que o banco aumentou a sua competência técnica em benchmarking.

\section{Aumento da competência técnica do BB em pesquisa aplicada}

$\mathrm{O}$ indicador desse desempenho foi um conjunto de pesquisas criadas após o término do curso pelas turmas. Com relação à satisfação do cliente foram criadas três pesquisas: Cliente Pessoa Física em 1999, Pessoa Jurídica em 2001, e Governo em 2002. Além dessas pesquisas, pela primeira vez a empresa realizou, em 2001, a pesquisa de posicionamento institucional, onde são identificados os atributos de um banco mais desejados pelos clientes de cada segmento e o quanto a empresa apresenta esses atributos. A quantidade de pesquisas criadas e a relevância dos seus resultados mostram que as percepções individuais são confirmadas no nível organizacional.

\section{Aumento da base de clientes}

$\mathrm{O}$ aumento na base de clientes foi observado em termos de Pessoas Física e Jurídica. O crescimento no primeiro caso, de 1996 a 2002, foi da ordem de 119\%. Já o aumento na base de clientes Pessoa Jurídica, entre 1999 e 2002, foi de $21 \%$. No período estudado, os resultados indicam que o banco atraiu novos clientes, e os índices foram bastante significativos.

\section{Diminuição da perda de clientes}

Esse indicador destaca a capacidade de manter os clientes da empresa. De acordo com as entrevistas realizadas, o banco não tem um indicador preciso quanto à diminuição da perda de clientes.

Dos 12 indicadores de desempenho organizacionais investigados intensivamente, pelas entrevistas estruturadas, nove $(75 \%)$ confirmaram as percepções dos funcionários identificados pelos questionários.

\section{Outros fatores possivelmente relacionados aos resultados organizacionais}

Vale destacar que o alcance dos resultados apresentados teve a contribuição do curso de acordo com os principais intervenientes: treinados e seus superiores. Entretanto, esses resultados organizacionais não podem ser atribuídos somente ao treinamento. Essa pesquisa também tentou identificar outros fatores organizacionais que auxiliaram na produção desses resultados, na visão das principais unidades da empresa.

De acordo com as entrevistas, os fatores mais sig- nificativos foram: reestruturações organizacionais, que passaram a focalizar o cliente; desenvolvimento tecnológico, que permitiu avanços no auto-atendimento, a entrada na Internet e a criação de "sistemas clientes" mais eficazes; investimento na profissionalização dos funcionários; valorização da equipe e do desempenho, com a reformulação dos instrumentos de avaliação de desempenho; implantação da gestão por resultados; difusão dos direitos do consumidor dentro e fora da empresa; abertura do BB para conhecer outras empresas; chegada de novos funcionários, com postura mais voltada para o mercado; e melhorias no layout das agências.

De acordo com os entrevistados, houve um alinhamento de ações dentro da organização que possibilitou o alcance dos resultados citados. Esse alinhamento facilitou a compreensão das pessoas quanto aos rumos da organização e à importância da satisfação do cliente como diferencial competitivo.

A aquisição dos conceitos de marketing foi fundamental para promover a melhoria do desempenho individual e da organização, mas os resultados poderiam ser diferentes se as pessoas não tivessem encontrado o apoio necessário para aplicar o que aprenderam. Isso foi demonstrado no nível individual, com o resultado da análise de variância, que evidenciou relações significativas entre o suporte psicossocial e os efeitos do treinamento no nível dos indivíduos. Esse suporte pode ser visualizado em dois níveis: no local de trabalho, com o apoio dos superiores e colegas, e no nível da organização, com políticas, diretrizes e ações que reforçaram a importância dos conteúdos aprendidos.

Além dos resultados organizacionais mencionados, os entrevistados identificaram outros resultados ou projetos desenvolvidos em suas unidades que acreditam ter recebido contribuição significativa do curso. Eles foram agrupados em três grandes categorias: cultura de foco no cliente; criação de produtos e serviços personalizados para cada tipo de cliente; e ampliação de canais de relacionamento com o cliente. São impactos de treinamento verificados em amplitude e não em profundidade, como foram todos os outros anteriormente mencionados, nos níveis individual e organizacional.

\section{CONCLUSÃO}

Os resultados mostraram que o impacto do BB MBA Marketing para o desempenho no trabalho dos indiví- 
duos situou-se entre bom e ótimo em $74 \%$ dos itens avaliados. Esses conceitos fazem parte do sistema de classificação dos níveis de impacto utilizados pela empresa, e são baseados nas médias de impacto obtidas em cada desempenho específico que foi aperfeiçoado pelo treinamento. O impacto na organização obteve os conceitos bom e ótimo para $96 \%$ dos desempenhos organizacionais. Em relação ao percentual anterior, esse incremento pode ter acontecido porque havia itens na avaliação de impacto no trabalho que se referiam a desempenhos específicos de determinadas áreas, não fazendo parte das atividades de todos os participantes do curso. No nível da organização, mesmo não sendo atribuição do funcionário treinado, há uma percepção geral do que pode ser atribuído ao treinamento. De qualquer forma, é um resultado curioso, pois de acordo com o modelo de avaliação proposto por Kirkpatrick (1976), ou com a abordagem multinível (Klein e Kozlowski, 2000), no nível organizacional seriam esperados impactos menores ou no máximo iguais aos esperados no nível individual.

É importante resgatar os motivos que deram origem à formulação do BB MBA Marketing: "melhorar a técnica de marketing, com a aproximação de conhecimento, no nível conceitual, técnico e operacional; e criar um ambiente de apoio para o marketing profissional, pessoas com conteúdos semelhantes, e com linguagem comum, que apóiam as decisões". Esses motivos ressaltam a necessidade de aprimoramento técnico no tema e capacidade de assessorar decisões baseada no marketing. Os resultados mostrados ao longo deste artigo confirmam que os maiores impactos estão na melhoria da competência técnica em marketing em vários aspectos, e que essa melhoria se refletiu em resultados organizacionais significativos.

No entanto, a principal contribuição pretendida neste trabalho não foi na área de marketing, mas lançar luz sobre as possibilidades metodológicas para a investigação do impacto de treinamento no nível organizacional. Para tanto, é essencial considerar o aspecto temporal. Avaliar o impacto de treinamento na organização alguns meses após o término do curso seria ineficaz, pois o processo de produção dos resultados é por emersão (começa no indivíduo treinado, propaga-se pelos grupos ou unidades dos quais ele faz parte e se concretiza em resultados organizacionais). Para Klein e Kozlowski (2000), esse processo é lento, e esse aspecto precisa ser considerado no desenho de pesquisa. Além disso, esse intervalo de tempo pode variar em função da natureza das com- petências individuais e das mudanças organizacionais esperadas do treinamento.

Ao realizar essa investigação, é fundamental considerar o uso de diversas fontes de informação para facilitar a identificação dos propósitos da organização ao desenvolver o treinamento. Essas fontes podem ser instrutores e planejadores instrucionais, treinandos, seus superiores, executivos das áreas que demandaram o treinamento, material didático e documentos. Além disso, os procedimentos de coleta de dados para a avaliação do treinamento podem variar, utilizandose de entrevistas individuais ou em grupos, questionários e pesquisa documental. A abordagem pode ser intensiva, tentando encontrar singularidades, ou extensiva, tentando encontrar regularidades, ou ainda incorporar elementos dessas duas abordagens, como ocorreu no presente trabalho. A vantagem de ter realizado uma abordagem intensiva, após uma extensiva, foi mapear previamente onde estariam localizados os impactos, antes de lançar a empreitada de realizar entrevistas e posteriormente buscar os indicadores organizacionais nelas identificados.

Outro aspecto relevante é a natureza da medida de impacto de treinamento no nível organizacional. Mesmo que a identificação de resultados organizacionais seja iniciada a partir de percepções dos indivíduos treinados, é necessário encontrar indicadores no nível da organização que dêem suporte a essas percepções. Se isso não for feito, o que se terá como resultado será uma avaliação da percepção dos indivíduos sobre o impacto de treinamento na organização e não evidências do impacto em si.

De qualquer forma, todos esses indicadores, nos dois níveis investigados, são sempre de atribuição de impacto e não evidências incontestáveis de que sem o treinamento tais efeitos não ocorreriam. Ou que somente com o treinamento seria possível ter obtido tais efeitos. Para produzir esse segundo tipo de evidência seria necessário utilizar o método de desenho experimental, com pré e pós-testes, e grupos controle e experimental, algo impensável numa pesquisa de campo. Assim, foi possível identificar as prováveis contribuições do treinamento, mas não foi possível isolar o efeito de outras variáveis. Essa falta de controle do desenho de pesquisa pode ser uma outra explicação para o fato curioso, e que contraria referenciais teóricos consagrados, de que teria ocorrido maior impacto no nível organizacional do que no nível individual. Também não foi objetivo mensurar o grau de contribuição do treinamento para os resultados organizacio- 
nais, comparada a outros fatores, finalidade legítima e que deveria ser perseguida no futuro para permitir avanços teóricos.

Para resolver esses dilemas metodológicos, a solução adotada foi identificar a contribuição do treinamento para os resultados da empresa, descrevendo também outros fatores organizacionais que se alinharam para a geração desses resultados. Dessa forma, está se considerando que treinamento é um dos fatores responsáveis pela geração de resultados. Outros fatores de contexto organizacional podem contribuir ou restringir o grau em que as pessoas utilizam o que aprenderam em treinamento, como tem sido demonstrado nas pesquisas sobre impacto de treinamento no trabalho e foi corroborado nesta pesquisa. Os resultados do estudo intensivo ainda sugerem que, em termos de impacto no nível organizacional, pode existir uma variedade de fatores de contexto que facilita o alcance dos resultados, tais como a estratégia e estrutura organizacionais, o modelo de gestão, a gestão de recursos humanos e a tecnologia. Parece que todos esses fatores precisam estar alinhados com os conteúdos e objetivos do treinamento para que ele possa gerar plenamente os efeitos desejados pela organização.

As limitações deste estudo são de diversas ordens, e algumas delas já foram mencionadas nos parágrafos anteriores. Mas há outras. Uma delas é que os resultados apresentados não podem ser generalizados para toda a população de funcionários treinados porque o número de respostas válidas (87) foi inferior à quantidade mínima necessária (132).

A segunda limitação está ligada ao fato de que, de acordo com os respondentes, os indicadores identificados se restringiram aos 10 maiores impactos no desempenho da organização. Outros resultados poderiam ser agregados se todos os 26 itens tivessem sido considerados nas entrevistas, objetivo a ser perseguido em futuras pesquisas.

Uma terceira limitação está relacionada à não concepção a priori de um modelo teórico para predizer impacto de treinamento no desempenho dos indivíduos nem da organização. O objetivo foi conceber um método para a mensuração dos efeitos de treinamento no nível da organização, em função da carência encontrada na literatura.

No futuro, o avanço metodológico perseguido por essa pesquisa deve ser acompanhado de um avanço teórico: poderiam ser pesquisados quais fatores organizacionais são fundamentais para promover melhores índices de impacto de treinamento nas organiza- ções, incluindo ainda fatores extra-organizacionais que afetam as empresas e seu desempenho nos contextos sociais, culturais, políticos e econômicos. Além disso, poderiam ser envidados esforços para a realização de pesquisas com desenhos experimentais e quase experimentais.

\section{REFERÊNCIAS BIBLIOGRÁFICAS}

ABBAD, G. Um modelo integrado de avaliação do impacto de treinamento no trabalho-IMPACT. 1999. 262 p. Tese (Doutorado em Psicologia) - Universidade de Brasília, Brasília, 1999.

ABBAD, G.; PILATI, R.; PANTOJA, M. J. Avaliação de treinamento: análise da literatura e agenda de pesquisa. RAUSP - Revista de Administração, v. 38 , n. 3, p. 205-218, 2003.

ÁVILA, A. F. D.; BORGES-ANDRADE, J. E., IRIAS, L. J. M.; QUIRINO, T. R. Formação do capital humano e retorno dos investimentos em treinamento na Embrapa. Série Documentos D R H Embrapa, Brasília, v. 4, p. 170. 1983.

BIRDI, K. The bigger picture: identifying the factors influencing training effectiveness. Trabalho apresentado no seminário Working to Learn, Sheffield, 2000. Não publicado.

BORGES-ANDRADE, J. E. Avaliação somativa de sistemas instrucionais: integração de três propostas. Tecnologia Educacional, v. XI, n. 46, p. 29-39, 1982

BORGES-ANDRADE, J. E.; SIRI, C. Impacts of training: the PM\&E Project's training. In: HORTON, D.; MACKAY, R.; ANDERSEN, A.; DUPLEICH, L. (Orgs.) Evaluating capacity development in planning, monitoring, and evaluation: a case from agricultural research. The Hague: International Service for National Agricultural Research, Study n. 3, p. 1-56, 2000. CD-ROM.

BORGES-ANDRADE, J. E. Desenvolvimento de medidas em avaliação de treinamento. Estudos de Psicologia (UFRN), v. 7, n. Especial, p. 31-43, 2002.

BROAD, M. L.; NEWSTROM, J. W. Transfer of training: action-packed strategies to ensure high payoff from training investments. Boston, MA: Addison-Wesley, 1992

KIRKPATRICK, D. L. Evaluation of training. In: CRAIG, R. L. (Org.), Training and development handbook. New York: McGraw Hill, 1976. p. 87112.

KLEIN, K. J.; KOZLOWSKI, S. W. J. Multilevel theory, research, and methods in organizations: foundations, extensions and new directions. San Francisco: Jossey-Bass, 2000 
LACERDA, E. R. M.; ABBAD, G. Impacto de treinamento no trabalho: investigando variáveis motivacionais e organizacionais como suas preditoras. RAC - Revista de Administração Contemporânea, v. 7, n. 4, p. 77-96, 2003.

MENESES, P. P. M.; ABBAD, G. Preditores individuais e situacionais de auto e heteroavaliação de impacto de treinamento no trabalho. RAC - Revista de Administração Contemporânea, v. 7, edição especial, p. 185-204, 2003

NOE, R. A.; SCHMITT, N. The influence of trainee attitudes on training effectiveness: test of a model. Personnel Psychology, v. 39, n. 3, p. 497-523, 1986.

PHILIPS, J. J. Handbook of training evaluation and measurement methods. Houston: Gulf, 1997.
PUENTE-PALACIOS, K. Depender ou não depender, eis a questão: um estudo multinível do efeito de padrões de interdependência na satisfação dos membros das equipes de trabalho. In: ENCONTRO ANUAL DA ASSOCIAÇÃO NACIONAL DOS PROGRAMAS DE PÓS-GRADUAÇÃO EM ADMINISTRAÇÃO, 27, 2003, Atibaia. Anais...Atibaia: Anpad, 2003. CD-ROM.

ROUILliER, J. Z.; GOLDSTEIN, I. L. The relationship between organizational transfer climate and positive transfer of training. Human Resource Development Quarterly, v. 4, n. 4, p. 377-390, 1993.

SALAS, E.; CANNON-BOWERS, J. The science of training: a decade of progress. Annual Review of Psychology, v. 52, p. 471-499, 2001.

SALLORENZO, L. H. Avaliação de impacto de treinamento no trabalho: analisando e comparando modelos de predição, 2000. 69 p. Dissertação (Mestrado em Psicologia) - Universidade de Brasília, Brasília, 2000.

\section{Artigo recebido em 29.10.2003. Aprovado em 19.04.2004.}

\section{Isa Aparecida de Freitas}

Doutoranda em Psicologia Social e Trabalho pela Universidade de Brasília. Pesquisadora Sênior da Universidade Corporativa Banco do Brasil. Interesses de pesquisa em planejamento e avaliação de treinamento, aprendizagem em organizações, avaliação de potencial e de desempenho, orientação profissional, políticas inovadoras de gestão de pessoas e qualidade de vida no trabalho.

E-mail: isaafreitas@aol.com

Endereço: AOS 01, Bloco F, apto. 411 - Brasília - DF, 70660-016.

\section{Jairo Eduardo Borges-Andrade}

Professor Titular do Departamento de Psicologia Social e do Trabalho do Instituto de Psicologia da UnB. M. Sc. e Ph. D. em Sistemas Instrucionais pela Florida State University. Pesquisador 1-A e coordenador de grupo no CNPq. Interesses de pesquisa em treinamento e desenvolvimento de pessoal, comportamento organizacional e pesquisa em psicologia organizacional.

E-mail: jeborges@linkexpress.com.br

Endereço: SQN 106, Bloco I, apto. 605 - Brasília - DF, 70742-090. 